\title{
Synthesis and Spectroscopic Characterization of Water-Soluble Fluorescent Ag Nanoclusters
}

\author{
Chengzhi Zheng, Huiping Wang, Lingzhi Liu, Manjun Zhang, \\ Jiangong Liang, and Heyou Han
}

State Key Laboratory of Agricultural Microbiology, College of Science, Institute of Chemical Biology,

Huazhong Agricultural University, Wuhan 430070, China

Correspondence should be addressed to Jiangong Liang; liangjg@mail.hzau.edu.cn

Received 25 March 2013; Accepted 25 April 2013

Academic Editor: Zhi-Ke He

Copyright ( $(2013$ Chengzhi Zheng et al. This is an open access article distributed under the Creative Commons Attribution License, which permits unrestricted use, distribution, and reproduction in any medium, provided the original work is properly cited.

\begin{abstract}
Water-soluble fluorescent Ag nanoclusters (NCs) were synthesized at room temperature with sodium dodecyl sulfonate (SDS) as a protective agent. The effects of synthetic conditions on the fluorescence properties of Ag NCs were investigated. The results show that the fluorescence intensity of Ag NCs strongly depends on the synthetic conditions, such as the molar ratio of $\mathrm{AgNO}_{3}$ versus SDS and sodium borohydride $\left(\mathrm{NaBH}_{4}\right)$, the reaction time, and the $\mathrm{pH}$ value of the reaction solution. Under the optimum conditions, the as-prepared Ag NCs exist in face-centered-cubic phase with an average size of $2 \mathrm{~nm}$. Fluorescence spectra of Ag NCs show emission peaks at $365 \mathrm{~nm}$ for different excitation wavelength. Resonant absorptions are observed at $203 \mathrm{~nm}$ and $277 \mathrm{~nm}$ in the absorption spectrum, which can be used to establish the electronic levels in the Ag NCs system.
\end{abstract}

\section{Introduction}

In recent years, noble metal nanoclusters have received considerable attention due to their marvelous optical, physical, and electrical properties for use in sensing, biological imaging, and single-molecular spectroscopy [1-7]. Among the various noble metal clusters reported to date, lownuclearity Ag NCs have been the subject of intense interest due to their low toxicity, ultrasmall size $(<2 \mathrm{~nm})$, and good fluorescence properties $[8,9]$. In the past years, several synthetic strategies have been proposed for the preparation of Ag NCs such as radiolytic [10, 11], photochemical [12], sonochemical [13], and chemical reduction approaches in the presence of various scaffolds, including polyelectrolyte [11], dendrimers [14], peptides [15], and DNA [16]. However, these methods usually involve complex processes or require expensive raw materials.

In the present work, we reported a facile one-pot synthetic route to prepare water-soluble fluorescent Ag NCs with the help of SDS. Compared with the previous methods using DNA, polymers, or peptides as templates, SDS, as a common commercial surfactant, is much simpler and more economical.

\section{Experimental Section}

2.1. Synthesis of $A g N C s$. In a typical experiment, a freshly prepared mixture solution of $\mathrm{AgNO}_{3}(1.0 \mathrm{mM})$ and SDS $(50 \mu \mathrm{M})$ was incubated in the ice bath for $c a .40 \mathrm{~min}$ and then reduced by the addition of $\mathrm{NaBH}_{4}(2.0 \mathrm{mM})$ with vigorous stirring for another $3 \mathrm{~h}$ at room temperature. The $\mathrm{pH}$ value of the reaction solution was adjusted by acetate buffer solution.

2.2. Characterization. The UV-Vis absorption spectrum was obtained in the range of $190-700 \mathrm{~nm}$, with $1.0 \mathrm{~cm} \times 1.0 \mathrm{~cm}$ quartz cuvette on a Thermo Nicolet Corp model Evolution 300 (America). All fluorescence spectra were recorded by a Shimadzu RF-5301PC Spectrofluorometer (Japan) equipped with a $20 \mathrm{~kW}$ xenon discharge lamp as a light source. The size and morphology of Ag NCs were acquired using a FEI Tecnai G20 transmission electron microscope (TEM) with 


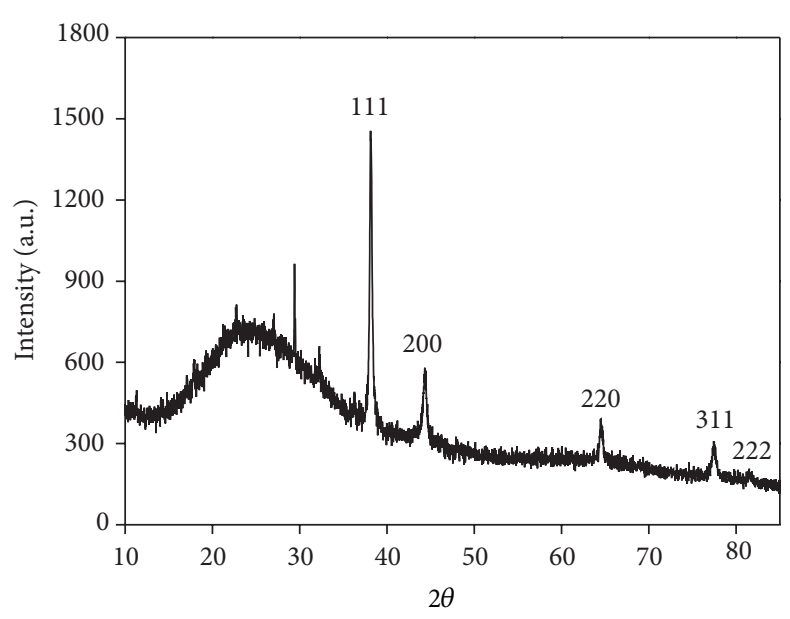

(a)

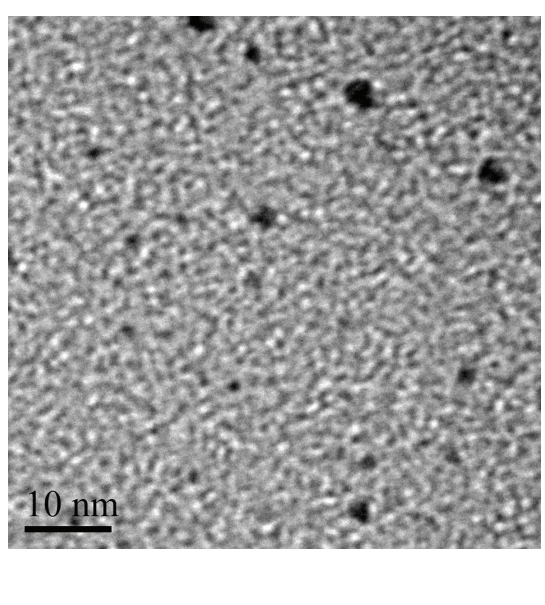

(b)

FIGURE 1: (a) The XRD pattern and (b) TEM image of Ag NCs.

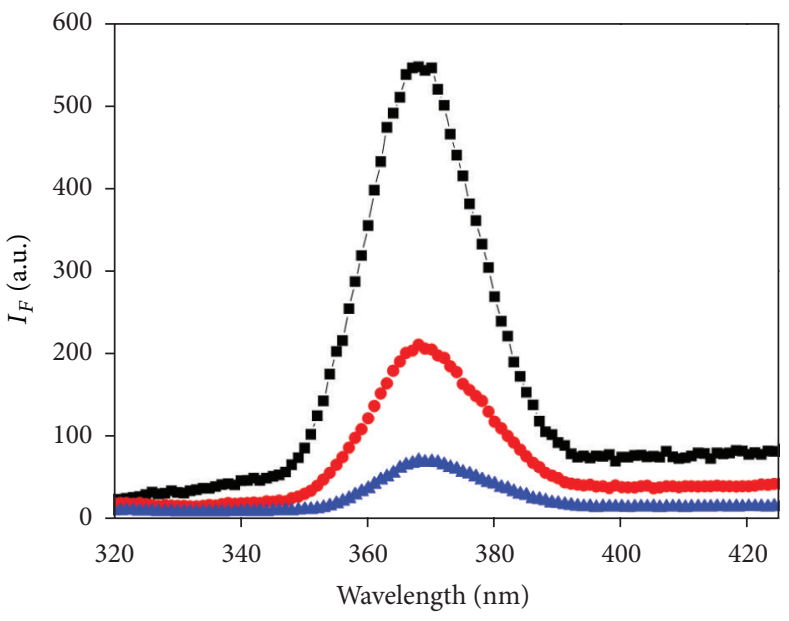

(a)

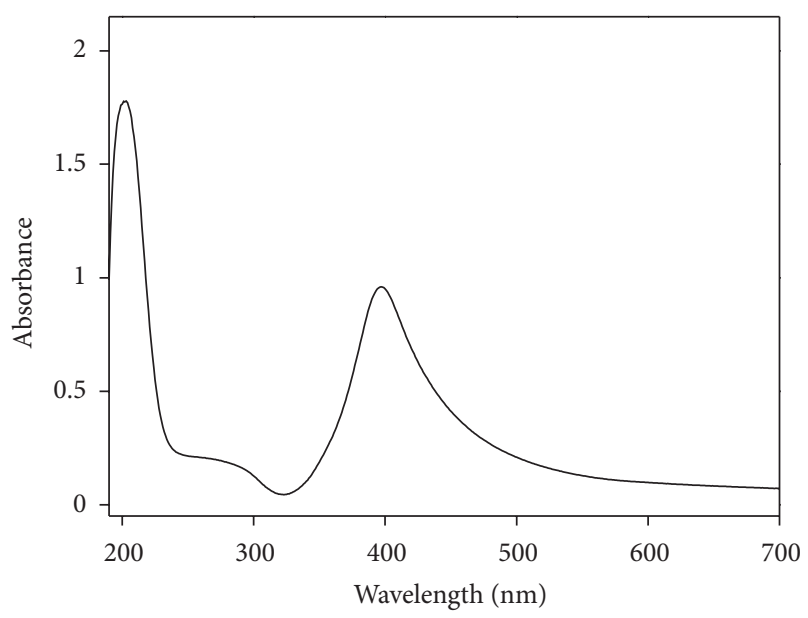

(b)

Figure 2: (a) Fluorescence emission spectra of Ag NCs at $\lambda_{\text {ex }}=220 \mathrm{~nm}$ (black curve), $240 \mathrm{~nm}$ (red curve), and $260 \mathrm{~nm}$ (blue curve), respectively; (b) UV-visible absorption spectrum of Ag NCs.

an acceleration voltage of $200 \mathrm{kV}$. Powder X-ray diffraction (XRD) was recorded using a D/Max-3B X-ray diffractometer (Rigaku International Corp., Japan) with $\mathrm{Cu} \mathrm{K}_{\alpha}$ source (40 kV and $30 \mathrm{~mA}$ ). All pH measurements were tested with a Model MP120 pH meter (Mettler-Toledo Instruments Ltd., Swiss).

\section{Results and Discussion}

A typical XRD pattern of Ag NCs is shown in Figure 1(a). It is seen that the major peaks locate at $2 \theta$ values of $38.12^{\circ}, 44.28^{\circ}$, $64.43^{\circ}, 77.48^{\circ}$, and $81.54^{\circ}$, which correspond to (111), (200), (220), (311), and (222) planes, respectively [17]. The peaks position and relative intensity are in good agreement with the values of $\mathrm{Ag}$ in the standard card (JCPDS number 04-783) and reveal that Ag NCs are in face-centred-cubic structure.
The corresponding TEM image (Figure 1(b)) indicates that the average size of Ag NCs is about $2 \mathrm{~nm}$ and the nanoclusters are spherical in shape.

Figure 2(a) manifests the fluorescence spectra of Ag NCs. Compared with the reported fluorescence spectra of Ag NCs that contain several emission peaks under different excitation wavelengths [18], the maximal emission of our Ag NCs is only observed at $365 \mathrm{~nm}$ with excitation wavelength $\left(\lambda_{\mathrm{ex}}\right)$ in the range of 220-260 nm. Assuming that one cluster has only one main specific emission peak [19], we can infer that there is only one cluster in our synthesized Ag NCs. The quantum yield (QY) of Ag NCs was estimated by using quinoline sulfate as a reference sample $[15,20]$. The QY of as-prepared Ag NCs is $0.84 \%$, which is comparable with the QY of Ag NCs reported in the reference $[21,22]$. Figure 2(b) shows the UVVis spectrum of Ag NCs. An apparent peak at $397 \mathrm{~nm}$ was 


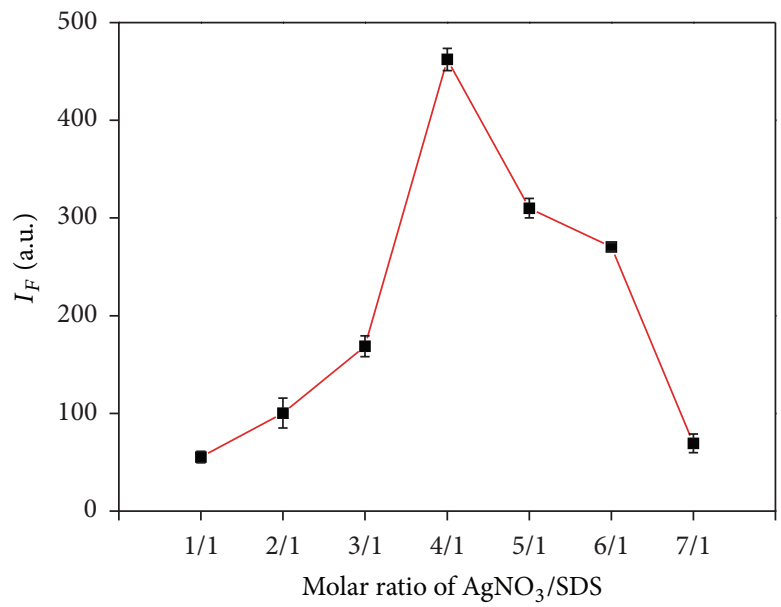

(a)

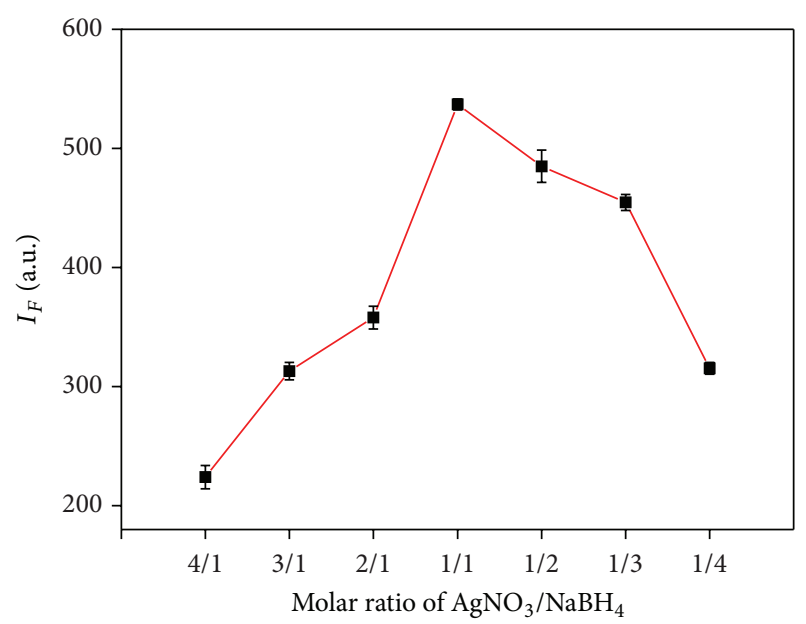

(b)

Figure 3: The effect of molar ratio of (a) $\mathrm{AgNO}_{3} / \mathrm{SDS}$ and (b) $\mathrm{AgNO}_{3} / \mathrm{NaBH}_{4}$ on the fluorescence intensity of $\mathrm{Ag} \mathrm{NCs}$.

observed in the spectrum, and it can be assigned to surface plasmons resonance arising from collective oscillations of the valence electrons in the electromagnetic field of the incident light. There are two absorption peaks at $203 \mathrm{~nm}$ and $277 \mathrm{~nm}$, respectively, which are consistent with the result reported by Siwach and coworkers [23]. The two absorption peaks can be ascribed to resonant peaks. Fluorescence from the noble metal has been well studied and mainly assigned to transitions of the electrons between the conduction band below the Fermi level and holes in the d bands [24]. A detailed study of fluorescence properties of the metal nanoclusters [25] allows us to ascribe this to transitions involving more than one excited state. The fluorescent behavior of Ag NCs observed is summarized in Scheme 1. The peaks at $277 \mathrm{~nm}$ and $203 \mathrm{~nm}$ correspond to the transitions between the ground state, the first excited state, and the second excited state. The only way fluorescence emission at $365 \mathrm{~nm}$ from both these two absorption peaks can be achieved is through an internal conversion involving these excited states.

The effects of synthetic conditions on the fluorescence properties of Ag NCs are very important and should be optimized carefully. Firstly, the influence of the molar ratio of $\mathrm{AgNO}_{3}$ versus SDS and $\mathrm{NaBH}_{4}$ on the fluorescence intensity of Ag NCs was studied. Figure 3(a) shows the effects of molar ratio of $\mathrm{AgNO}_{3}$ versus SDS on fluorescence intensity of $\mathrm{Ag}$ $\mathrm{NCs}$ at $365 \mathrm{~nm}$ wavelength. And the maximum intensity was acquired when the molar ratio was 4:1. From Figure 3(b), we can also find that when the molar ratio of $\mathrm{AgNO}_{3}$ versus $\mathrm{NaBH}_{4}$ was 1:1, Ag NCs adopt the maximum fluorescence intensity.

We also investigated the effect of the reaction time and $\mathrm{pH}$ values on the fluorescence intensity of as-prepared $\mathrm{Ag}$ NCs. As shown in Figure 4(a), the optimum reaction time is $3 \mathrm{~h}$. Figure $4(\mathrm{~b})$ indicates the effects of $\mathrm{pH}$ values on the fluorescence intensity. The maximum intensity was obtained at $\mathrm{pH} 4.0$, and the nanocluster synthesized at either higher or lower $\mathrm{pH}$ value showed weaker fluorescence intensity. At higher $\mathrm{pH}$, the hydroxide ions will compete with sulfonate

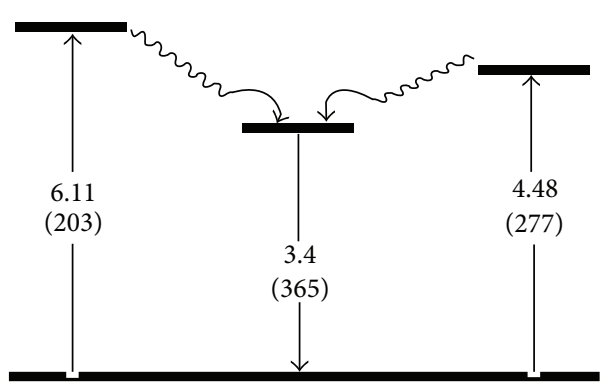

Scheme 1: Schematic diagram of energy levels explaining the fluorescence emission spectrum observed for Ag NCs. Energy values are given in $\mathrm{eV}$ (and $\mathrm{nm}$ inside parenthesis).

ions for the complexing of silver ions and even formed silver oxide. At lower $\mathrm{pH}$, the existence of hydrogen bond will weaken the interaction between sulfonic acid groups and silver atoms. In our experiment, the optimum $\mathrm{pH}$ value that balances the influence of both factors in the SDS-assisted synthesis of Ag NCs was found to be 4.0.

The synthetic process of Ag NCs mainly involves two steps. Firstly, $\mathrm{Ag}^{+}$was reduced to $\mathrm{Ag}$ sol by $\mathrm{NaBH}_{4}$ and immediately was coordinated with borohydride ions. Then, sulfonic acid groups substituted the borohydride ions and then formed Ag NCs, since they have a better affinity for metal atoms. We expect SDS to be an ideal template for at least three reasons: (1) SDS carries sulfonic acid groups capable of coordinating with $\mathrm{Ag}^{+}$; (2) SDS exhibits necessary hydrophobicity due to the presence of dodecyl groups, and previous studies found that hydrophobic regions facilitated the formation of metal nanoclusters [1]; (3) most importantly, SDS as protective agent is much more simpler and economical.

\section{Conclusion}

A convenient chemical reduction method for the synthesis of water-soluble fluorescent Ag NCs has been successfully 


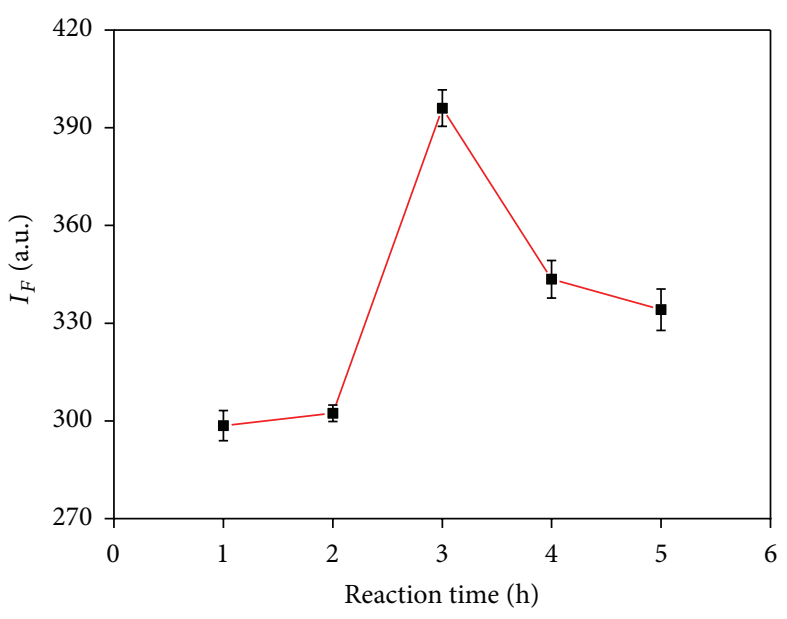

(a)

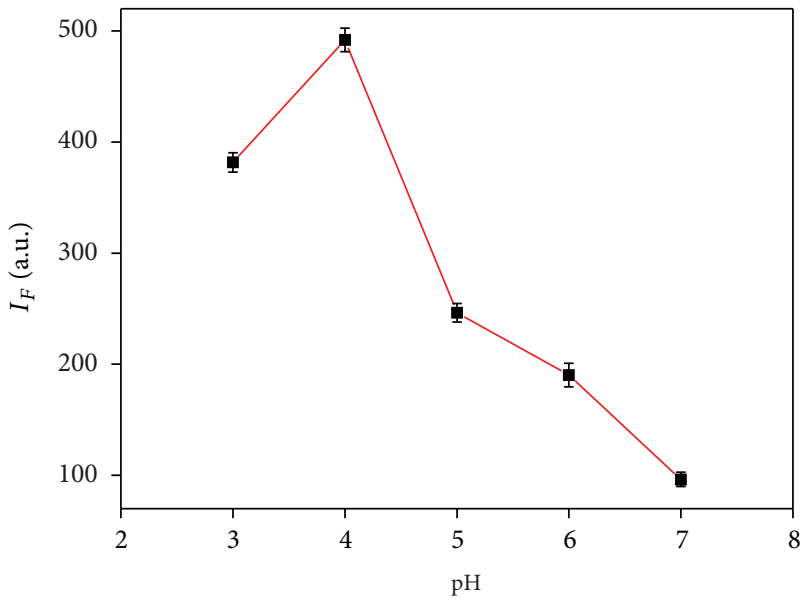

(b)

Figure 4: The effect of (a) reaction time and (b) $\mathrm{pH}$ value on the fluorescence intensity of Ag NCs.

developed. The ease of synthesis as well as the utilization of SDS makes these inexpensive and fluorescent Ag NCs attractive due to their good reproducibility and great potential for large-scale synthesis. Furthermore, the stability and excellent fluorescent properties of these Ag NCs enable them to find widespread applications in bioimaging, chemical, and biosensing.

\section{Acknowledgments}

This work was supported by National Nature Science Foundation of China (20905028, 21205043), the Fundamental Research Funds for the Central Universities (2011PY009, 2011QC021), and Huazhong Agricultural University Scientific \& Technological Self-Innovation Foundation (2010SC035).

\section{References}

[1] J. Yu, S. A. Patel, and R. M. Dickson, "In vitro and intracellular production of peptide-encapsulated fluorescent silver nanoclusters," Angewandte Chemie International Edition, vol. 46, no. 12, pp. 2028-2030, 2007.

[2] Y. C. Shiang, C. C. Huang, and H. T. Chang, "Gold nanodotbased luminescent sensor for the detection of hydrogen peroxide and glucose," Chemical Communications, no. 23, pp. 34373439, 2009.

[3] L. Shang, S. Brandholt, F. Stockmar, V. Trouillet, M. Bruns, and G. U. Nienhaus, "Effect of protein adsorption on the fluorescence of ultrasmall gold nanoclusters," Small, vol. 8, no. 5, pp. 661-665, 2012.

[4] Y. Wang, J. Zhang, L. Huang et al., "Novel application of Ag nanoclusters in fluorescent imaging of human serum proteins after native polyacrylamide gel electrophoresis (PAGE)," Chemistry European Journal, vol. 18, no. 5, pp. 1432-1437, 2012.

[5] X. Yuan, Y. Tay, X. Dou, Z. Luo, D. T. Leong, and J. Xie, "Glutathione-protected silver nanoclusters as cysteine-selective fluorometric and colorimetric probe," Analytical Chemistry, vol. 85, no. 3, pp. 1913-1919, 2013.
[6] L. Shang, R. M. Dorlich, V. Trouillet, M. Bruns, and G. U. Nienhaus, "Ultrasmall fluorescent silver nanoclusters: protein adsorption and its effects on cellular responses," Nano Research, vol. 5, no. 8, pp. 531-542, 2012.

[7] S. I. Tanaka, J. Miyazaki, D. K. Tiwari, T. Jin, and Y. Inouye, "Fluorescent platinum nanoclusters: synthesis, purification, characterization, and application to bioimaging," Angewandte Chemie International Edition, vol. 50, no. 2, pp. 431-435, 2011.

[8] H. Xu and K. S. Suslick, "Water-soluble fluorescent silver nanoclusters," Advanced Materials, vol. 22, no. 10, pp. 1078-1082, 2010.

[9] C. Guo and J. Irudayaraj, "Fluorescent Ag clusters via a proteindirected approach as a $\mathrm{Hg}(\mathrm{II})$ ion sensor," Analytical Chemistry, vol. 83, no. 8, pp. 2883-2889, 2011.

[10] B. Adhikari and A. Banerjee, "Short-peptide-based hydrogel: a template for the in situ synthesis of fluorescent silver nanoclusters by using sunlight," Chemistry European Journal, vol. 16, no. 46, pp. 13698-13705, 2010.

[11] L. Shang and S. Dong, "Facile preparation of water-soluble fluorescent silver nanoclusters using a polyelectrolyte template," Chemical Communications, no. 9, pp. 1088-1090, 2008.

[12] J. Zhang, S. Xu, and E. Kumacheva, "Photogeneration of fluorescent silver nanoclusters in polymer microgels," Advanced Materials, vol. 17, no. 19, pp. 2336-2340, 2005.

[13] H. Xu and K. S. Suslick, "Sonochemical synthesis of highly fluorescent Ag nanoclusters," ACS Nano, vol. 4, no. 6, pp. 3209$3214,2010$.

[14] J. Zheng and R. M. Dickson, "Individual water-soluble dendrimer-encapsulated silver nanodot fluorescence," Journal of the American Chemical Society, vol. 124, no. 47, pp. 1398213983, 2002.

[15] Y. Cui, Y. Wang, R. Liu et al., "Serial silver clusters biomineralized by one peptide," ACS Nano, vol. 5, no. 11, pp. 8684-8689, 2011.

[16] G. Y. Lan, W. Y. Chen, and H. T. Chang, "One-pot synthesis of fluorescent oligonucleotide Ag nanoclusters for specific and sensitive detection of DNA," Biosensors and Bioelectronics, vol. 26, no. 5, pp. 2431-2435, 2011. 
[17] X. Gao, L. Wei, H. Yan, and B. Xu, "Green synthesis and characteristic of core-shell structure silver/starch nanoparticles," Materials Letters, vol. 65, no. 19-20, pp. 2963-2965, 2011.

[18] T. Udaya and T. Pradeep, "Luminescent $\mathrm{Ag}_{7}$ and $\mathrm{Ag}_{8}$ clusters by interfacial synthesis," Angewandte Chemie International Edition, vol. 49, no. 23, pp. 3925-3929, 2010.

[19] J. Zheng, C. Zhang, and R. M. Dickson, "Highly fluorescent, water-soluble, size-tunable gold quantum dots," Physical Review Letters, vol. 93, no. 7, pp. 077402-4, 2004.

[20] T. Zhou, M. Rong, Z. Cai, C. Yang, and X. Chen, "Sonochemical synthesis of highly fluorescent glutathione-stabilized Ag nanoclusters and $\mathrm{S}^{2-}$ sensing," Nanoscale, vol. 4, no. 14, pp. 41034106, 2012.

[21] J. Sharma, H. C. Yeh, H. Yoo, J. H. Werner, and J. S. Martinez, "A complementary palette of fluorescent silver nanoclusters," Chemical Communications, vol. 46, no. 19, pp. 3280-3282, 2010.

[22] B. Adhikari and A. Banerjee, "Facile synthesis of water-soluble fluorescent silver nanoclusters and HgII sensing," Chemistry of Materials, vol. 22, no. 15, pp. 4364-4371, 2010.

[23] O. P. Siwach and P. Sen, "Synthesis and fluorescence properties of Ag nanoparticles," Solid State Communications, vol. 148, no. 5-6, pp. 221-225, 2008.

[24] G. T. Boyd, Z. H. Yu, and Y. R. Shen, "Photoinduced luminescence from the noble metals and its enhancement on roughened surfaces," Physical Review B, vol. 33, no. 12, pp. 7923-7936, 1986.

[25] A. Ledo, F. Martínez, M. A. López-Quintela, and J. Rivas, "Synthesis of Ag clusters in microemulsions: a time-resolved UV-vis and fluorescence spectroscopy study," Physica B, vol. 398 , no. 2, pp. 273-277, 2007. 

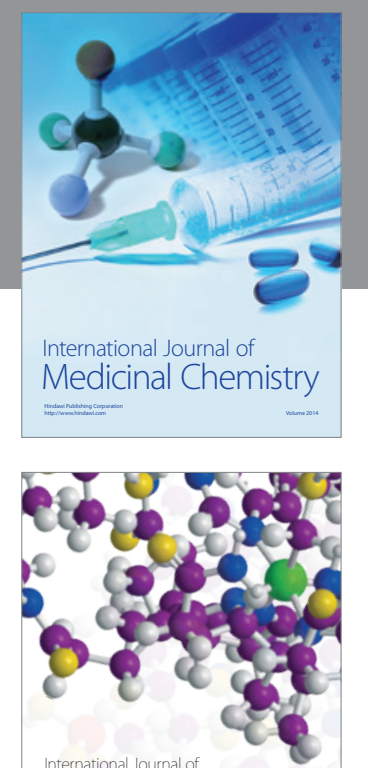

\section{Carbohydrate} Chemistry

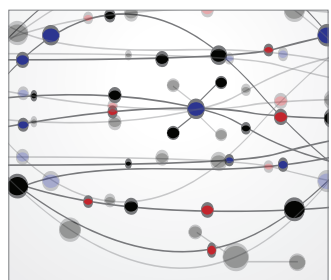

The Scientific World Journal
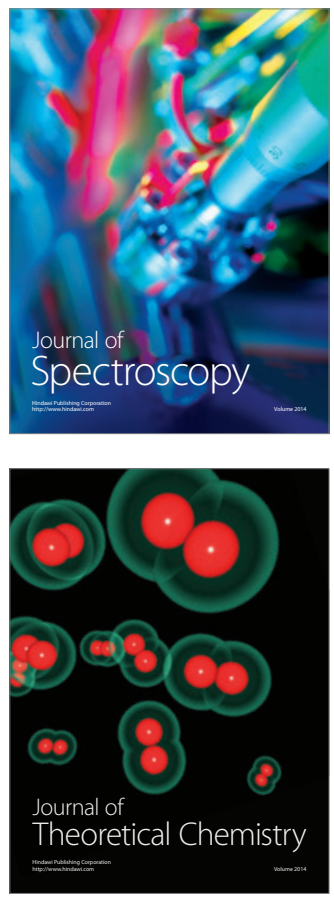
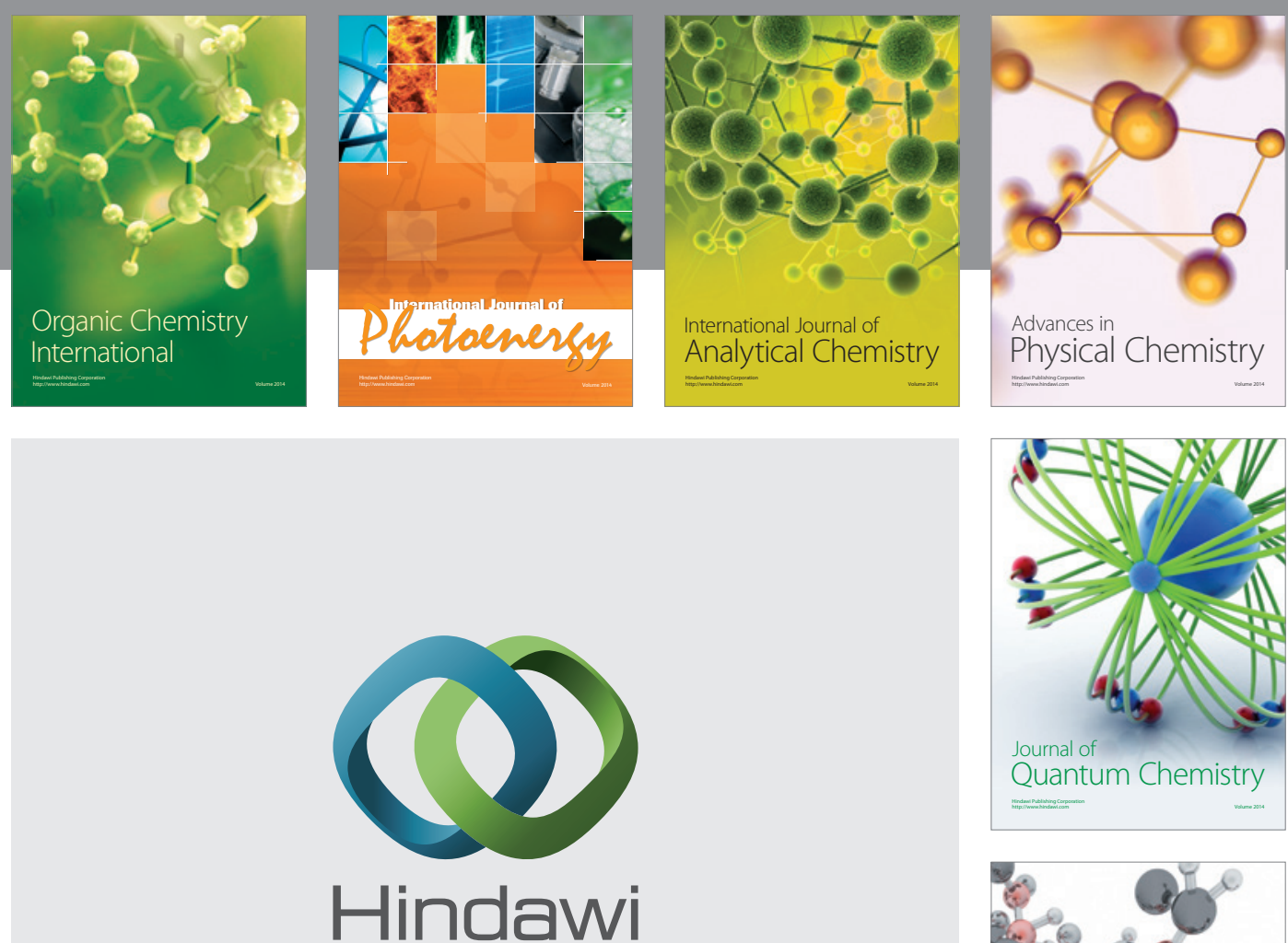

Submit your manuscripts at

http://www.hindawi.com

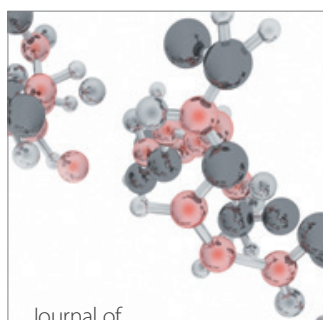

Analytical Methods

in Chemistry

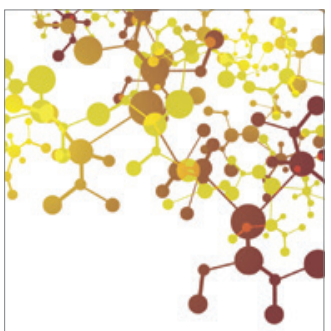

Journal of

Applied Chemistry

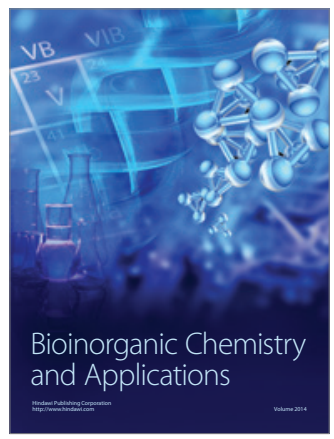

Inorganic Chemistry
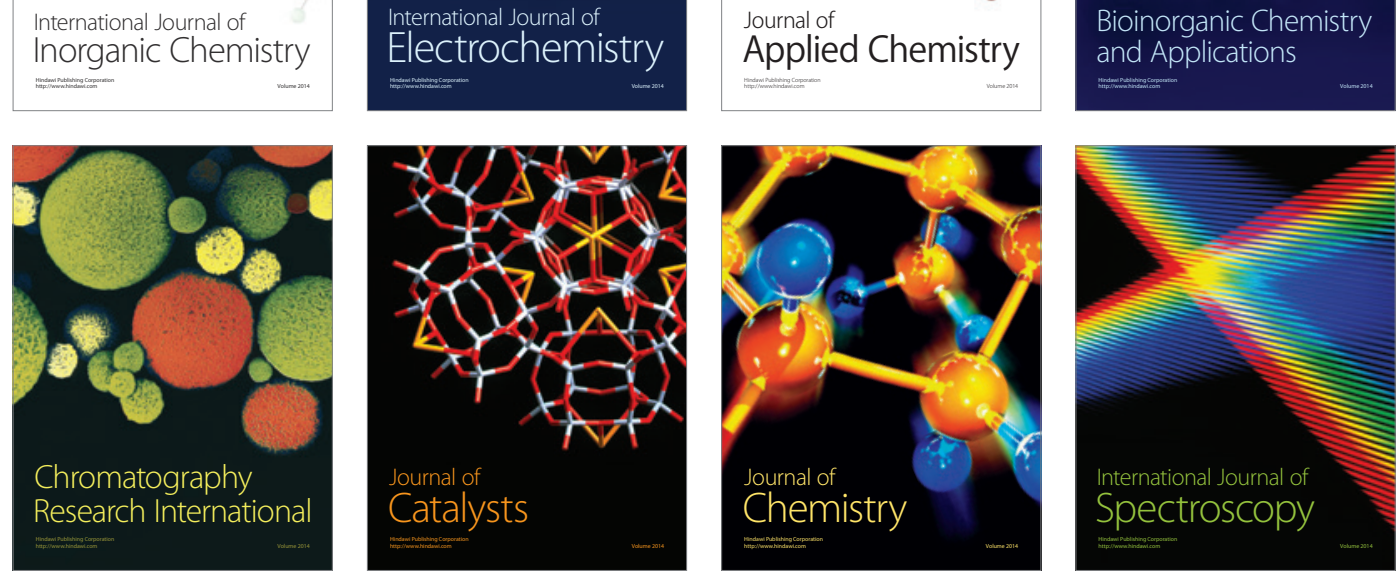